\title{
Le système d'innocuité vaccinale canadien en huit étapes : des notions pour les travailleurs de la santé
}

\author{
Noni E. MacDonald, Barbara J. Law \\ Société canadienne de pédiatrie, comité des maladies infectieuses et d'immunisation, Ottawa (Ontario) \\ Correspondance : Société canadienne de pédiatrie, 100-2305, boul. St. Laurent, Ottawa (Ontario) K1G 4J8 \\ Courriel : info@cps.ca; site Web : www.cps.ca
}

Pour lire le texte intégral du présent document de principes, rendez-vous à la page https://academic.oup.com/pch/

issue $/ 22 / 4$

\section{Résumé}

Parce qu'ils s'inquiètent de l'innocuité vaccinale, certains parents hésitent à faire vacciner leurs enfants. Les professionnels de la santé jouent un rôle déterminant auprès des parents pour leur faire comprendre que le Canada est un chef de file en matière d'innocuité vaccinale. Le présent point de pratique fournit une mise à jour des huit étapes du système d'innocuité vaccinale canadien : 1) une analyse pré-homologation et un processus d'approbation fondés sur des données probantes; 2) une réglementation rigoureuse pour les fabricants; 3 ) des recommandations indépendantes et fondées sur des données probantes à l'égard de l'utilisation des vaccins; 4) la formation et les normes sur les compétences en matière d'immunisation pour les travailleurs de la santé; 5) des programmes de pharmacovigilance pour déceler et 6) déterminer la causalité des effets secondaires suivant l'immunisation (ESSI); 7) un programme pour la détection des signaux relatifs à l'innocuité et à l'efficacité vaccinales; et 8) les cliniques de vaccination spéciales du Réseau de recherche canadien sur l'immunisation pour les enfants qui ont été victimes de graves ESSI.

Mots-clés : $\quad$ AEFI; BGTD; CAEFISS; IMPACT; NACI; Vaccine safety

\section{Remerciements}

Le comité de la pédiatrie communautaire de la Société canadienne de pédiatrie a révisé le présent point de pratique.

\section{COMITÉ DES MALADIES INFECTIEUSES ET D'IMMUNISATION DE LA SCP}

Membres : Natalie A. Bridger MD; Shalini Desai MD; Jane C. Finlay MD (membre sortante); Ruth Grimes MD (représentante du conseil); Charles P. S. Hui MD (membre sortant); Timothy Mailman MD; Joan L. Robinson MD (présidente); Marina Salvadori MD (membre sortante); Otto G. Vanderkooi MD

Représentants : Upton D. Allen MBBS, Groupe de recherche canadien sur le sida chez les enfants; Tobey Audcent MD, Comité consultatif de la médecine tropicale et de la médecine des voyages, Agence de la santé publique du Canada; Carrie Byington MD, comité des maladies infectieuses, American Academy of Pediatrics; Fahamia Koudra MD, Le Collège des médecins de famille du Canada; Nicole Le Saux MD, Programme canadien de surveillance de l'immunisation (IMPACT); Rhonda Kropp B. Sc. inf. MHP, Agence de la santé publique du Canada; Jane McDonald MD, Association pour la microbiologie médicale et l'infectiologie Canada; Dorothy L. Moore MD, Comité consultatif national de l'immunisation

Conseillère : Noni E. MacDonald MD

Auteures principales : Noni E. MacDonald MD, Barbara J. Law MD 